\title{
Light scattering of human red blood cells during metabolic remodeling of the membrane
}

\author{
YongKeun Park, ${ }^{\mathrm{a}, \mathrm{b}, *}$ Catherine A. Best-Popescu, ${ }^{\mathrm{c}}$ Ramachandra R. Dasari, ${ }^{\mathrm{a}}$ and Gabriel Popescu \\ aMassachusetts Institute of Technology, G. R. Harrison Spectroscopy Laboratory, 77 Massachusetts Avenue, Cambridge, \\ Massachusetts 02139 \\ bHarvard-MIT Division of Health Science and Technology, 77 Massachusetts Avenue, Cambridge, Massachusetts 02139 \\ ' University of Illinois at Urbana-Champaign, College of Medicine, Urbana, 405 North Mathews Avenue, Urbana, \\ Illinois 61801 \\ dUniversity of Illinois at Urbana-Champaign, Beckman Institute for Advanced Science and Technology, Department of \\ Electrical and Computer Engineering, 405 North Mathews Avenue, Urbana, Illinois 61801
}

\begin{abstract}
We present the light scattering properties of individual human red blood cells (RBCs). We show that both the RBC static and dynamic scattering signals are altered by adenosine $5^{\prime}$-triphosphate (ATP)-driven membrane metabolic remodeling. To measure the light scattering signal from individual RBCs, we use diffraction phase microscopy together with a Fourier transform light scattering technique. RBC cytosolic ATPs are both chemically and metabolically depleted, and the corresponding scattering signals are compared with the light scattering signal of normal RBCs having physiologic levels of ATP. $\odot 2011$ Society of Photo-Optical Instrumentation Engineers (SPIE). [DOI: 10.1117/1.3524509]
\end{abstract}

Keywords: light scattering; red blood cells; erythrocyte; quantitative phase microscopy.

Paper 10205SSR received Apr. 18, 2010; revised manuscript received Sep. 17, 2010; accepted for publication Oct. 8, 2010; published online Jan. 27, 2011.

\section{Introduction}

The red blood cell (RBC) membrane cortex consists of two coupled membranes: a lipid bilayer and a $2-\mathrm{D}$ spectrin network. ${ }^{1}$ The lipid bilayer provides bending rigidity, and the spectrin network gives shear resistance. The cytoplasm of RBC, mainly consisting of viscous hemoglobin (Hb) solution, offers damping property that dissipates energy. The RBC membrane cortex has unique viscoelastic properties that play a crucial role in $\mathrm{RBC}$ survival and blood rheology. Altered RBC membrane elasticity has been implicated in early RBC cell death and in the pathophysiology of a number of disorders of microcirculation. ${ }^{2}$

This RBC membrane is remarkably soft and elastic, and thus exhibits dynamic fluctuations, observed as "flickering," with amplitudes of the order of tens of nanometers. These fluctuations have been explored extensively to better understand the biophysical properties of the RBC membrane and related pathophysiological conditions. ${ }^{3-12}$ First observed a century ago, ${ }^{13}$ its origin is generally believed to stem from thermal forces. ${ }^{3,4}$ Different interference-based optical microscopic techniques have been employed to measure membrane fluctuations. ${ }^{4,6,12}$ The mechanical properties of the RBC membranes have been studied by assuming that the membrane fluctuations are purely driven by thermal energy and thus follow Brownian dynamics. ${ }^{4,6}$ In a recent report, no relation between adenosine $5^{\prime}$-triphosphate (ATP) and membrane fluctuations was observed when the outer edge of RBCs was probed. ${ }^{14}$ In contrast, a correlation between ATO concentration in $\mathrm{RBC}$ cytosol and the membrane fluctuation

*Current affiliation: Department of Physics, Korean Advanced Institute of Science and Technology, Daejeon, Republic of Korea 305-701.

Address all correspondence to: YongKeun Park, Department of Physics, Korean Advanced Institute of Science and Technology, Daejeon, Republic of Korea 305701; E-mail: yk.park@kaist.ac.kr. amplitude was reported when the local averaged fluctuations of RBC membranes were measured. ${ }^{15}$ Furthermore, the full-field dynamic fluctuations in RBC membranes have also been systemically studied in the presence and absence of ATP. These studies showed that the membrane cortex is not a static structure, but undergoes dynamic remodeling depending on ATP. ${ }^{16}$ In the presence of ATP, the membrane fluctuations showed the enhanced fluctuation over the convex regions on the RBC. This may result from the continuous binding and unbinding between the lipid bilayer and spectrin network. ${ }^{16}$ This metabolic remodeling of the RBC membrane results in altered mechanical properties. ${ }^{17,18}$ This remodeling is also related to maintaining the characteristic biconcave shape of RBC. ${ }^{16}$ In the absence of ATP, the RBC membrane loses its biconcave shape and undergoes the morphological transformation to echinocyte. ${ }^{19}$ The dynamic molecular interactions between the lipid bilayer and the spectrin network remain to be elucidated.

Light scattering techniques have proven useful in studying RBCs. Static light scattering of RBCs is commonly used for measuring the cell size and hemoglobin concentration in flow cytometry. ${ }^{20}$ Dynamic light scattering has been utilized for studying the RBC membrane dynamics. ${ }^{21,22}$ Recently, both static and dynamic light scattering of RBCs were used to distinguish healthy RBC and malaria-parasite invaded RBCs. ${ }^{23}$ Dynamic light scattering signals can provide quantitative information about the biomechanical properties of the RBC membrane. ${ }^{23}$ Light scattering spectroscopy has unique advantages over direct observation of the specimen at the image plane. ${ }^{24}$ The optical resolution is limited by diffraction in direct imaging, whereas the light scattering signal remains sensitive to changes much smaller than the wavelength of the light. Also, in angle-resolved elastic light scattering, the scattering signals

1083-3668/2011/16(1)/011013/7/\$25.00 @ 2011 SPIE 
are expressed in terms of scattering angle, which can be readily related to the size of scatters. In contrast, in the 2-D imaging map, scatters in different sizes are spread over 2-D spatial coordinates, which makes it difficult to quantitatively compare samples.

Here, we study the effects of ATP on the RBC membrane by measuring the Fourier transform light scattering (FTLS) light scattering signal. FTLS is a novel experimental approach that uses quantitative phase imaging to perform light scattering studies on inhomogeneous and dynamic media. ${ }^{25-29}$ To retrieve elastic light scattering signals from the RBCs, we implemented FTLS using diffraction phase microscopy (DPM) as the quantitative phase imaging method. ${ }^{27,30}$ We present both static and dynamic light scattering signals of RBCs following a low- and high-dose ATP depletion regimen over time. We first studied the static light scattering signals for normal and ATP-depleted RBCs. Then we compared the dynamic light scattering of normal and ATP-depleted RBCs. To study the reversibility of the ATP effects, we also studied the alterations in the membrane mechanical properties for the RBCs in which ATP was metabolically depleted and then repleted (returned to the cell medium for subsequent RBC uptake).

\section{Materials and Methods}

\subsection{Sample Preparation of Red Blood Cells}

Human RBCs from a healthy volunteer were collected in vacutainer tubes containing an anticoagulant (ethylenediaminetetraacetic acid). The tubes of blood were immediately centrifuged at $2000 \mathrm{~g}$ for $10 \mathrm{~min}$ at $10^{\circ} \mathrm{C}$ to separate $\mathrm{RBCs}$ from the plasma. The RBCs were then washed three times with phosphatebuffered saline (PBS). After washing, the RBCs were resuspended in PBS to approximately $20 \%$ hematocrit $(\mathrm{Ht})$.

We prepared six different groups of RBCs in this study following the standard protocol ${ }^{31}: 1$. RBCs with physiological levels of ATP (no additional treatment after resuspending RBC samples), 2. metabolically ATP-depleted RBCs, 3. irreversibly ATP-depleted RBCs (with high dose of inosine and iodoacetamide), 4. irreversibly ATP-depleted RBCs (with low dose of inosine and iodoacetamide), 5. ATP-repleted RBCs after metabolic ATP depletion, and 6. an osmotic control group. For metabolically ATP-depleted RBCs, the RBCs were incubated in glucose-free PBS for $24 \mathrm{~h}$ at $37^{\circ} \mathrm{C}$. We prepared two different subgroups for the irreversible ATP depletion (high dose and low dose). For the first irreversibly ATP-depleted group (highdose depletion group), the RBCs were incubated without glucose, in the presence of 10-mM inosine (A3221, Sigma-Aldrich, Saint Louis, Missouri) and 6-mM iodoacetamide (I1024, SigmaAldrich), for $2 \mathrm{~h}$ at $20^{\circ} \mathrm{C}$. For the second group of irreversibly ATP-depleted RBCs (low-dose depletion group), the RBCs were treated the same as the high-dose ATP-depleted group except that 3-mM inosine and 1-mM iodoacetamide was used. Inosine consumes ATP and iodoacetamide blocks ATP production by inhibiting glyceraldehydes-3phosdehydrogenase. For the ATPreintroduced RBCs, $10 \mathrm{mM}$ of D-glucose was added to the RBC suspension in the metabolically ATP-depleted group. For the osmotic control group, 10-mM L-glucose was added to a RBC suspension in the metabolically ATP-depleted group. This study was approved by the Massachusetts Institute of Technology's Committee on the Use of Humans as Experimental Subjects.

\subsection{Diffraction Phase Microscopy}

An $\operatorname{Ar}^{2+}$ laser $(\lambda=514 \mathrm{~nm})$ was used as illumination source for an inverted microscope (IX71, Olympus America Incorporated, Center Valley, Pennsylvania). The microscope was equipped with a $40 \times$ objective lens $(0.75 \mathrm{NA})$ that facilitates a diffraction-limited transverse resolution of about $400 \mathrm{~nm}$. With the additional relay optics used outside the microscope, the overall magnification of the system was approximately $200 \times$. An electron-multiplying charge-coupled device (EMCCD, Photonmax 512B, Princeton Instruments, Incorporated, Trenton, New Jersey) was used to image the interferogram. DPM employs the principle of laser interferometry in a common-path geometry and thus provides full-field quantitative phase images of RBCs with unprecedented optical path-length stability. The DPM optical path-length stability is $2.4 \mathrm{mrad}$, which corresponds to the displacement in RBC membrane of $3.3 \mathrm{~nm} .{ }^{32}$

Using DPM, the amplitude and phase of the complex image field associated with individual RBCs can be quantitatively measured with high precision, $U(x, y ; t)=A(x, y ; t) e^{j \phi(x, y ; t)}$, where $A(x, y ; t)$ is the amplitude and $\phi(x, y ; t)$ is the phase at a position of $(x, y)$ at time $t$. The refractive index difference $\Delta n$ between the RBC and the surrounding medium is mainly attributed to homogeneously distributed $\mathrm{Hb}$ protein in $\mathrm{RBC}$ cytosol. Thus, topographies of RBCs can be directly obtained from the measured phase image as $h(x, y ; t)=\lambda /(2 \pi \cdot \Delta n) \phi(x, y ; t)$, where $\lambda$ is the wavelength of the laser. We have used the values for $\Delta n$ measured from tomographic phase microscopy. ${ }^{33}$ The details of DPM instrumentation can be found elsewhere. ${ }^{30,32}$ Recently, DPM has been extended for measuring refractive index in the deep ultraviolet range and dispersion phase image. ${ }^{34,35}$

\subsection{Fourier Transform Light Scattering Technique}

In FTLS, the optical phase and amplitude of a coherent image field are quantified and propagated numerically to the scattering plane. ${ }^{27,36}$ By exploiting the efficient detection of the complex field at the image plane instead of the far-field plane, which fully utilizes the dynamic range of the detector and provides high angular resolution capabilities, FTLS provides highly accurate static and dynamic light scattering maps. Because it detects all the scattered angles (spatial frequencies) simultaneously in each point of the image, FTLS can be regarded as the spatial equivalent of Fourier transform infrared spectroscopy (FTIR), where all the temporal frequencies are detected at each moment in time.

To quantitatively measure the full-field maps of individual RBCs at the image plane, we employed DPM. ${ }^{30,32}$ To correct for the amplitude and phase shape of the illumination beam, the measured complex field image with sample was then divided by the complex field image without samples. From this corrected field, the far-field scattering intensity is calculated by using a 2-D (spatial) Fourier transform as

$$
I\left(q_{x}, q_{y} ; t\right)=\left|\frac{1}{2 \pi} \iint U(x, y ; t) e^{-j q_{x} x} e^{-j q_{y} y} d x d y\right|^{2},
$$

where $q_{x}, q_{y}$ are spatial frequencies defined as $q_{x}$ $=2 \pi n\left(\sin \theta_{x} / \lambda\right), q_{y}=2 \pi n\left(\sin \theta_{y} / \lambda\right)$, where $n$ is a refractive index of the medium. During Fourier transformation, we used 
the following normalization:

$\tilde{U}\left(q_{x}, q_{y}\right)=\frac{1}{\sqrt{2 \pi}} \frac{1}{\sqrt{2 \pi}} \iint U(x, y) e^{-j q_{x} x} e^{-j q_{y} y} d x d y$,

which was implemented in a 2-D fast Fourier transform (FFT) as

$$
\tilde{U}\left(q_{x}, q_{y}\right)=\frac{1}{\sqrt{N_{x}}} \frac{1}{\sqrt{N_{y}}} \sum_{x} \sum_{y} U(x, y) e^{-j q_{x} x} e^{-j q_{y} y},
$$

where the tilde denotes the Fourier transform pair and $N_{x}, N_{y}$ are the total pixel numbers along the $x$ and $y$ axes, respectively, such that the following equality for energy conservation (Parseval's theorem) is satisfied:

$$
\iint \tilde{U}\left(q_{x}, q_{y}\right) \tilde{U}^{*}\left(q_{x}, q_{y}\right) d q_{x} d q_{y}=\iint U(x, y) U^{*}(x, y) d x d y .
$$

The static scattering intensity map was measured as the time average of consecutive scattering intensity patterns, $\bar{I}\left(q_{x}, q_{y}\right)$ $=\left\langle I\left(q_{x}, q_{y} ; t\right)\right\rangle$. FTLS analysis was performed with the use of MATLAB (Math Works Inc., Natick, MA) and a custom-made script.

\subsection{Data Analysis}

$P$ values are calculated by two-tailed Mann-Whitney rank sum tests comparing light scattering signals between various test conditions. Error bars in the figures represent standard errors. All numbers following the \pm sign in the text are standard errors.

\section{Results and Discussion}

We first addressed the static light intensity scattering patterns of individual RBCs using FTLS. ${ }^{23}$ We measured the E-field of individual RBC samples at the image plane using DPM. The phase map in the E-field $\phi(x, y ; t)$ provides topological information about the $\operatorname{RBC} h(x, y ; t)$ (see Sec. 2.2). The time-averaged height maps of the RBCs $\bar{h}(x, y)=\langle h(x, y ; t)\rangle$ are shown in Figs. 1(a), 1(b), and 1(c). The characteristic biconcave shape of the RBC membrane [Fig. 1(a)] was lost when cytosolic ATP was irreversibly depleted from the RBC cytosol [Figs. 1(b) and 1(c)]. Thus, ATP is critical in maintaining the characteristic RBC biconcave shape. ${ }^{19}$ We then calculated the static light scattering patterns of RBCs $\bar{I}\left(q_{x}, q_{y}\right)$ from the measured E-field using a FTLS technique (see Sec. 2.3). The static light intensity patterns are plotted as a function of scattering angle from 0 to $48.6 \mathrm{deg}$ after azimuthal average and converting spatial frequency $q$ to angle $\theta$ by using the relation $q=2 \pi n \sin \theta / \lambda$ [Figs. 1(d), 1 (e), and 1(f)]. The scattering patterns of intact RBCs show the distinct periodic oscillatory features, which are consistent with previous measurements and simulations. ${ }^{20,37}$ The scattering patterns of ATP-depleted RBCs show periodic oscillations up to $15 \mathrm{deg}$.

To quantitatively compare the static light scattering patterns between intact RBCs and the two groups of the irreversibly ATPdepleted RBCs, we performed the two-tailed Mann-Whitney rank sum tests [Fig. $1(\mathrm{~g})]$. The averaged $p$-values from the fullforward scattering angle ( 0 to $48.6 \mathrm{deg}$ ) are larger than 0.1 for both groups of ATP-depleted groups when compared with the intact RBCs. However, averaged $p$-values for the low scattering angle range ( 0 to $10 \mathrm{deg}$ ) are $<0.1$ for both the high- and low-dose groups in the irreversible ATP-depletion groups compared to the normal RBCs. Static light scattering of RBCs is determined by the refractive index of cytoplasm and the cellular shape. As long as there is no significant net flux of water across the RBC membrane during ATP depletion, we can assume that there is no significant change of cytoplasmic refractive index during the depletion of ATP. Thus, the alterations to the static light scattering signal during ATP depletion is likely due to echinocytosis, the shape change from discocyte to echinocyte. The low $p$-values at the low scattering angles can be also be explained by echinocytosis, since it mainly causes morphological changes at large spatial scales (i.e., small scattering angle). The scattering light spectrums between high and low doses did not show a significant difference.

To address the dynamic light scattering signals with respect to the different levels of ATP, we measured the complex image field of RBCs for approximately 2 sec at 120 frames per sec. Then, we calculated the light scattering intensity patterns for each consecutive complex field map using a FTLS technique. Each scattering pattern at a specific time $t$ was calculated from the corresponding E-field map [Eq. (1)]. To quantitatively analyze the dynamic scattering signals, we calculated the normalized temporal autocorrelation functions from the dynamic light scattering intensity fluctuations $\langle I(\theta, \tau) I(\theta, 0)\rangle /\langle I(\theta, 0)\rangle^{2}$. The results are summarized in Figs. 2(a), 2(b), and 2(c).

The spectrum of the dynamic light scattering signal can be approximately described as Lorentzian, having a peak frequency $\omega_{0}$ and a line width $\Gamma$, and its autocorrelation is described as a damped cosine function: ${ }^{38}$

$$
G(\tau)=A+B \cos \left(\omega_{0} \tau+\varphi\right) e^{-\Gamma \tau} e^{-\beta^{2} \tau^{2}} .
$$

The phase term $\varphi$ is included to account for the deviation of the spectrum from an exact Lorentzian function, and the $\beta$ term represents the instrumental variations. For individual RBCs, the light scattering intensity autocorrelation was calculated at each scattering angle, from which $\omega_{0}$ and $\Gamma$ were retrieved by fitting to Eq. (5). Then, $\omega_{0}$ and $\Gamma$ are averaged from the specific range of low scattering angles ( 0 to $7 \mathrm{deg}$ ). The extracted values of $\Gamma$ are $2.91 \pm 0.49 \mathrm{~Hz}, 4.58 \pm 0.78 \mathrm{~Hz}$, and $4.41 \pm 0.61 \mathrm{~Hz}$ for the intact RBCs, the ATP-depleted RBCs with low dose, and the ATP-depleted RBCs with a high dose of inosine and iodoacetamide, respectively [Fig. 3(a)]. The values of $\Gamma$ for both ATPdepleted RBCs and the normal RBCs were significantly different ( $p<0.2$ for both high- and low-dose-treated RBCs). The extracted values of $\omega_{0}$ are $1.28 \pm 0.49,1.23 \pm 0.30$, and 2.26 $\pm 0.97 \mathrm{rad} / \mathrm{s}$ for the normal RBCs, the ATP-depleted RBCs with low dose, and the ATP-depleted RBCs with high dose, respectively [Fig. 3(b)]. Although the mean values of peak frequencies $\omega_{0}$ showed an increase for the irreversibly ATP-depleted group (high dose), the values for both ATP-depleted RBCs were not statistically different compared to the normal $\operatorname{RBC}(p>0.2)$. Close examination into both $\Gamma$ and $\omega_{0}$ after irreversibly depleting ATP with lose-dose depletion agents confers information about dose dependency. The values for $\Gamma$ increased with the lose dose of inosine and iodoacetamide, while the value for $\omega_{0}$ did not show a statistical difference compared to the normal RBC group. This result implies that the effective viscosity of the RBC membrane cortex is more sensitive to ATP levels than the elasticity. 

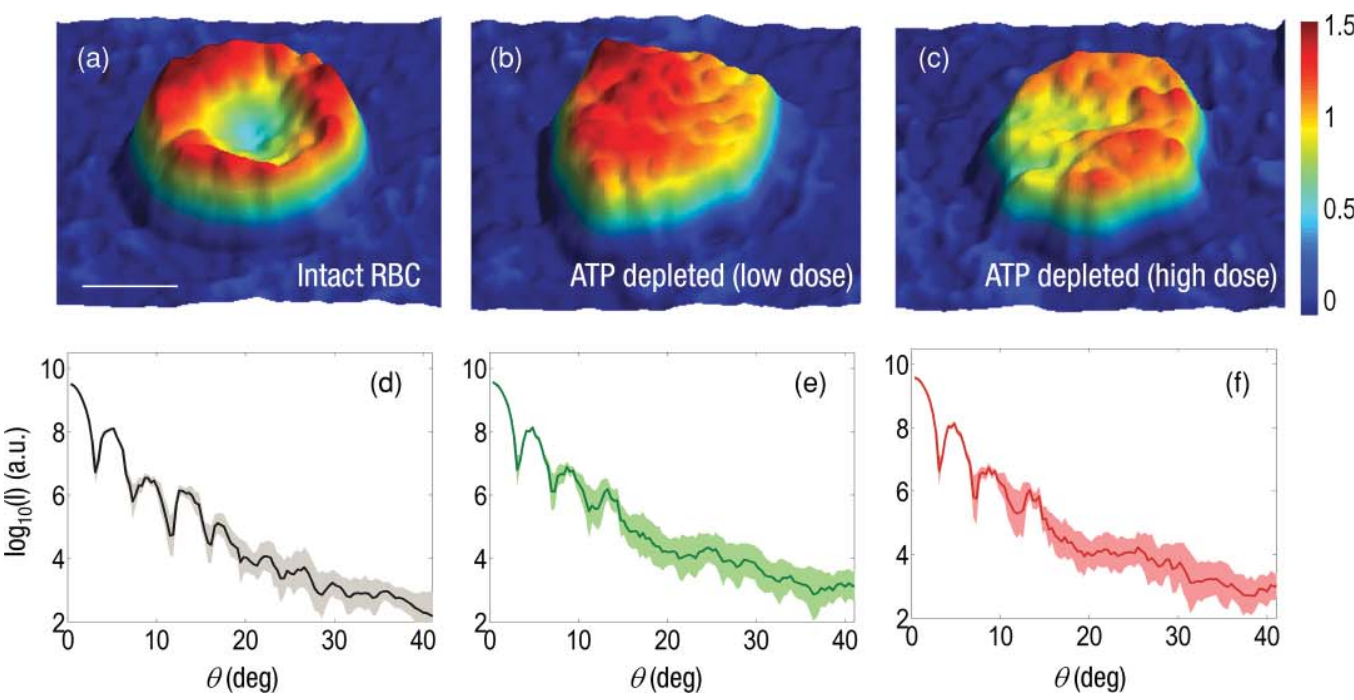

(g)

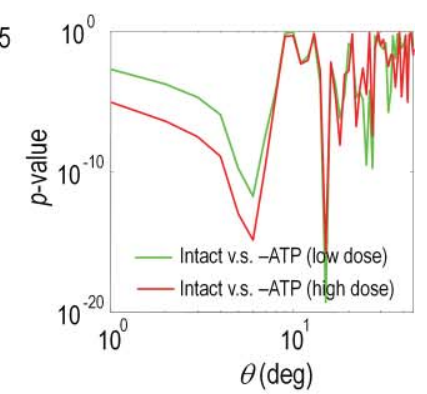

Fig. 1 The static scattering signal of RBC and the effects of ATP. Topographies of RBCs measured by diffraction phase microscopy: (a) a healthy RBC, (b) a RBC with irreversibly depleted ATP (low dose of inosine and iodoacetamide), and (c) high dose of inosine and iodoacetamide. Units for colorbar are micrometers. Scale bar indicates $5 \mu \mathrm{m}$. Light-intensity scattering patterns of (d) healthy RBCs, (e) RBCs with irreversibly depleted ATP (low dose of inosine and iodoacetamide), and ( $\mathrm{f}$ ) high dose of inosine and iodoacetamide. The thick line indicates the mean value and the area indicates the standard deviation ( $N=30 /$ group). (g) $p$-values of scattering patterns between healthy RBCs and different groups of RBCs. (Color online only.)

For a simple model where a flat lipid bilayer exhibits membrane fluctuations, dynamic light scattering can be directly used to retrieve the mechanical properties of the lipid; $\omega_{0}$ is directly related to membrane tension and $\Gamma$ to medium viscosity. ${ }^{38}$ However, the human RBC membrane has a complex biconcave shape, and there is no theoretical model to directly relate dynamic light scattering signals to the mechanical properties of the RBCs. Nonetheless, one can qualitatively relate the $\omega_{0}$ to the effective elasticity and $\Gamma$ to the effective viscosity of the RBC membrane cortex. $^{23}$

Our results show that after ATP-depletion with a high dose of inosine $(10 \mathrm{mM})$ and iodoacetamide $(6 \mathrm{mM})$, the dynamic light scattering signal exhibits increases in both $\Gamma$ and $\omega_{0}$ [Figs. 3(a) and 3(b)]. This increase in $\Gamma$ indicates that the effective viscosity of the RBC membrane cortex increases after ATP depletion. This is consistent with the previous report where the effective viscosity was retrieved by probing the power spectral density of membrane fluctuations at the rims of RBCs. ${ }^{17}$ The value for $\omega_{0}$ suggests an increase for the effective elasticity of the RBC after the depletion of ATP, which is also consistent with the previous report. ${ }^{17}$ The increase of the effective elasticity of the RBC membrane under ATP depletion can be explained by the increase of shear modulus of the spectrin network, bending modulus, and area expansion modulus of the lipid bilayer. ${ }^{17,18}$ The increase of the effective viscosity can be explained by the time-dependent imaginary part of the shear modulus of the spectrin network or the change in the dynamic binding and unbinding between the lipid layer or spectrin network. ${ }^{18}$ These changes in mechanical properties of RBC can be explained by structural or biochemical modifications of membrane-bound proteins depending on ATP level or shape changes in RBC morphology, or both.

To further study the effect of ATP level on RBC membrane properties, we addressed the reversibility in the dynamic light scattering signal. We introduced ATP to the metabolically ATP-depleted RBCs by adding D-glucose. D-glucose is transported into RBC cytosol and converted into ATP via glycolytic pathways. ${ }^{31}$ Then, we measured dynamic light scattering signals at several time points $(0,6$, and $24 \mathrm{~h}$ after reintroducing ATP).
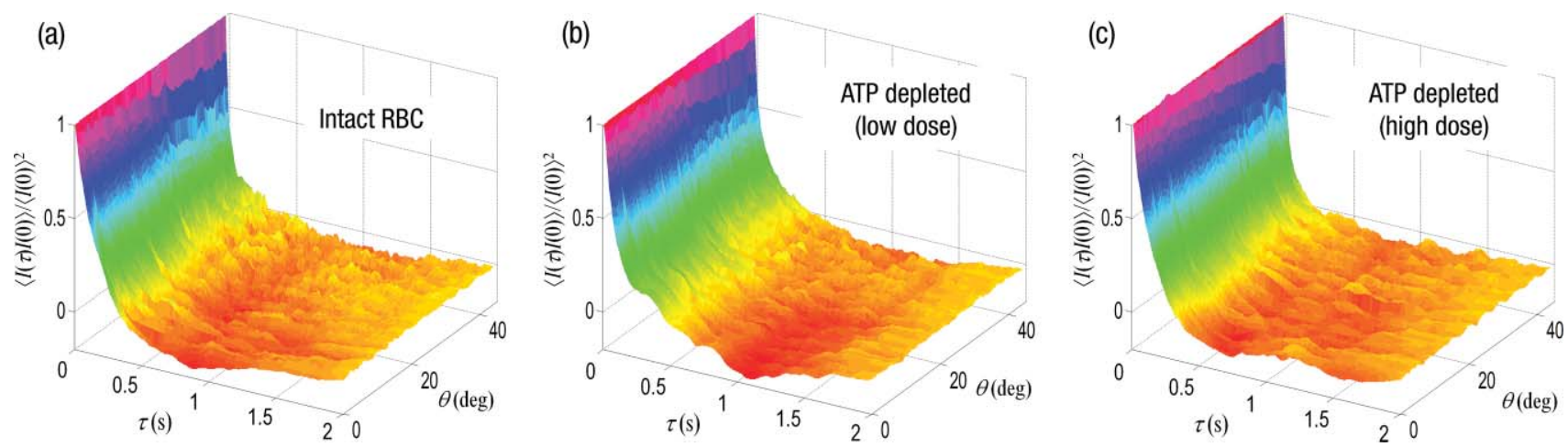

Fig. 2 Dynamic light scattering of RBCs with different cytosolic ATPs. Normalized temporal autocorrelations as a function of decay time and scattering angle of (a) healthy RBCs, (b) RBCs with irreversibly depleted ATP (low dose of inosine and iodoacetamide), and (c) high dose of inosine and iodoacetamide ( $N=30$ /group). 
(a)

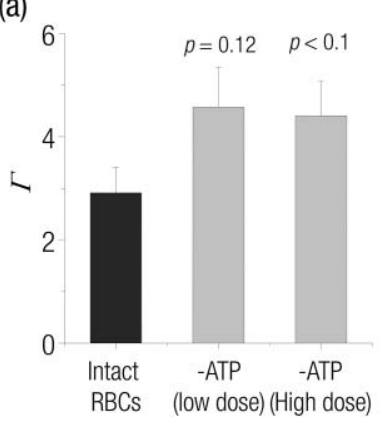

(b)

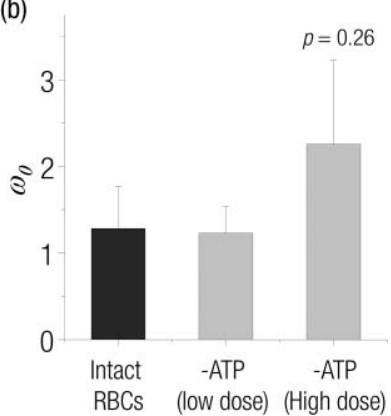

Fig. 3 (a) Line width $\Gamma$ and (b) peak frequency $\omega_{0}$ extracted from intact RBCs and RBCs with irreversibly depleted ATP (low and high doses of inosine and iodoacetamide). Error bars indicate the standard errors $(N=30 /$ group $)$.

The results are shown in Fig. 4. Following the same procedures described before, we retrieved both $\Gamma$ and $\omega_{0}$ for the metabolically ATP-depleted RBCs. Compared to the normal RBCs, $\Gamma$ increased to $4.41 \pm 0.56 \mathrm{~Hz}$ and $\omega_{0}$ increased to $1.85 \pm 0.44 \mathrm{~Hz}$ after ATP was metabolically consumed. These values for $\Gamma$ and $\omega_{0}$ for the metabolically ATP-depleted RBCs are very close to the irreversible ATP-depleted RBCs with a high dose (4.41 $\pm 0.61 \mathrm{~Hz}$ for $\Gamma$ and $2.26 \pm 0.97 \mathrm{rad} / \mathrm{s}$ for $\left.\omega_{0}\right)$. We also retrieved the values for $\Gamma$ and $\omega_{0}$ after reintroducing ATP to the metabolically ATP-depleted RBCs. Right after reintroduction of ATP, the value for $\Gamma$ decreased to $1.88 \pm 0.40 \mathrm{~Hz}$ and the value for $\omega_{0}$ decreased to $1.09 \pm 0.20 \mathrm{rad} / \mathrm{s}$. These values are close to the values for the normal RBCs $(2.91 \pm 0.49 \mathrm{~Hz}$ and $1.28 \pm 0.49 \mathrm{rad} / \mathrm{s}$ for $\Gamma$ and $\omega_{0}$, respectively). The values for both line widths and peak frequencies at $0 \mathrm{~h}$ after ATP reintroduction are not statistically different from the normal RBC group. This result shows that the alteration of mechanical properties of the RBC membrane cortex at depletion of ATP is a reversible process. The recovery of both $\Gamma$ and $\omega_{0}$ indicates that both elastic and viscous properties of the RBC membrane can be reversed following the reintroduction of ATP. To rule out the possibility that the observed alterations in the light scattering signal after reintroducing ATP were caused by osmotic effects of glucose, we prepared an osmotic control group in which L-glucose was added to a RBC suspension in the metabolically ATP-depleted group. Unlike D-glucose, L-glucose cannot be

utilized by RBC to produce ATP. The values for both $\Gamma$ and $\omega_{0}$ were not statistically different from the ones with the metabolically ATP-depleted RBCs. This result provides evidence that the alterations to light scattering signals are due to varying $\mathrm{RBC}$ cytosolic ATP levels.

To study the time-dependent effects of ATP depletion on the RBC membrane, we then measured the dynamic light scattering signals at 6 and $24 \mathrm{~h}$ post-D-glucose addition (Fig. 3). The values for $\Gamma$ increased from $1.88 \pm 0.40 \mathrm{~Hz}$ (at $0 \mathrm{~h}$ ) to $2.56 \pm 0.63 \mathrm{~Hz}$ and $4.69 \pm 1.08 \mathrm{~Hz}$ after 6 and $24 \mathrm{~h}$, respectively. The value for $\omega_{0}(1.09 \pm 0.16 \mathrm{rad} / \mathrm{s})$ after $6 \mathrm{~h}$ did not changed significantly from $1.09 \pm 0.20 \mathrm{rad} / \mathrm{s}$ (at $0 \mathrm{~h}$ after reintroducing ATP). The value for $\omega_{0}$ increased to $2.18 \pm 0.77 \mathrm{rad} / \mathrm{s}$ at $24 \mathrm{~h}$ after the reintroduction of ATP. The final values for both $\Gamma$ and $\omega_{0}$ became close to both the metabolically ATP-depleted group and the irreversible ATP-depleted group, whereas the mean value for $\Gamma$ increases continuously following the reintroduction of ATP. The mean value for $\omega_{0}$ changed very little during the first $6 \mathrm{~h}$, and then increased, reaching a value close to that of the metabolically deprived ATP group. However, the $p$-values for both $\Gamma$ and $\omega_{0}$ at $6 \mathrm{~h}$ after the reintroduction of ATP are not statistically different compared to the 0 -h time point. This result implies that there is a continuous transition in mechanical properties of the RBC membrane as cytosolic ATP is metabolically consumed.

In conclusion, using FTLS and DPM, we report both the static and dynamic light scattering signals of individual RBCs with different levels of ATP. Compared to conventional methods, this method can provide comprehensive quantitative information that consists of a quantitative phase imaging map and a simultaneous angular light scattering measurement. This enables us to selectively analyze individual RBCs, and provides both static and dynamic scattering signals. Thus, our measurements are free from statistical averaging and provide accurate light scattering characteristics of individual cells. The ATP-depleted RBCs show different static scattering signals compared with normal RBCs having physiological levels of ATP. The dynamic scattering results indicate that the alterations in mechanical properties of the RBC membranes are ATP dependent. ${ }^{17,18}$ Moreover, we show that the alterations in the $\mathrm{RBC}$ membrane mechanical properties are reversible processes. The approach in this study has many potential applications in studying RBC biomechanics. For example, the mechanical properties of the RBC membrane cortex can be studied in terms of cytosolic levels of ATP. We note (a) 8

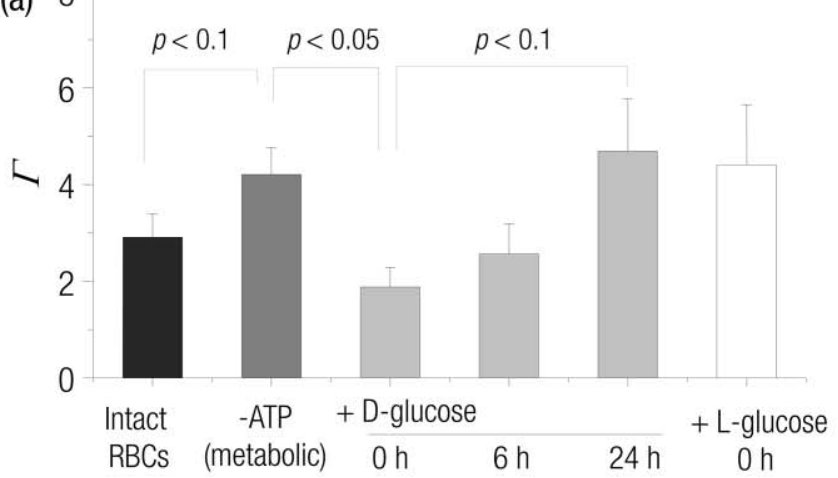

(b)

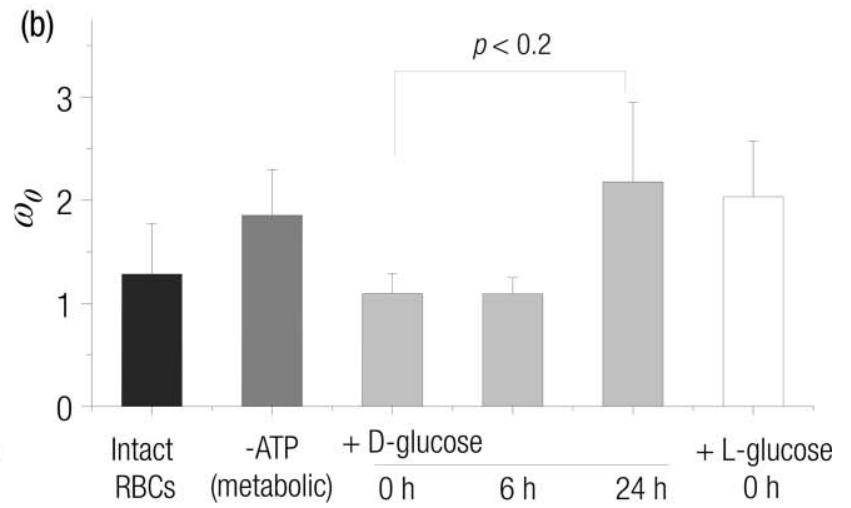

Fig. 4 (a) Line width $\Gamma$ and (b) peak frequency $\omega_{0}$ extracted from intact RBCs, RBCs with metabolically depleted ATP, RBCs with ATP was reintroduced $(+$ ATP), and ATP-reintroduced RBCs measured after 6 and 24 after adding ATP. Error bars indicate the standard errors $(\mathrm{N}=30 /$ group). 
that no external measurement of cytosol ATP was performed in this study. However, the cytosol ATP level can be measured by using an expression of recombinant targeted luciferases and bioluminescence imaging. ${ }^{39}$ The correlation between the quantified cytosolic ATP level and the alteration in the mechanical properties in the RBC membrane can provide useful information in understanding RBC membrane biophysics. The mechanical alterations in RBCs are complicated during physiologic flow, because ATP is released when the RBCs are subjected to shear stress during passage through the microvasculature. ${ }^{40}$ Unanswered questions surrounding RBC membrane biomechanics during circulation warrant further investigation in this field.

\section{Acknowledgment}

This research was supported by the National Institutes of Health (P41-RR02594-18-24). Author Park was supported by Samsung Scholarship. Author Popescu acknowledges support from the National Science Foundation (CAREER: 08-46660) and National Cancer Institute (R21: CA154813). The authors appreciate the guidance and advice from the late Michael S. Feld.

\section{References}

1. N. Mohandas and E. Evans, "Mechanical properties of the red bell membrane in relation to molecular structure and genetic defects," Ann. Rev. Biophys. Biomole. Struct. 23(1), 787-818 (1994).

2. S. Suresh, J. Spatz, J. P. Mills, A. Micoulet, M. Dao, C. T. Lim, M. Beil, and T. Seufferlein, "Connections between single-cell biomechanics and human disease states: gastrointestinal cancer and malaria," Acta Biomater. 1(1), 15-30 (2005).

3. F. Brochard and J. F. Lennon, "Frequency spectrum of the flicker phenomenon in erythrocytes," J. Physique 36, (1035-1047 (1975).

4. A. Zilker, H. Engelhardt, and E. Sackmann, "Dynamic reflection interference contrast (ric-) microscopy - a new method to study surface excitations of cells and to measure membrane bending elastic-moduli," J. Physique 48(12), 2139-2151 (1987).

5. N. Gov, A. G. Zilman, and S. Safran, "Cytoskeleton confinement and tension of red blood cell membranes," Phys. Rev. Lett. 90(22), 228101 (2003).

6. G. Popescu, T. Ikeda, K. Goda, C. A. Best-Popescu, M. Laposata, S. Manley, R. R. Dasari, K. Badizadegan, and M. S. Feld, "Optical measurement of cell membrane tension," Phys. Rev. Lett. 97(21), 218101 (2006).

7. G. Popescu, Y. K. Park, R. R. Dasari, K. Badizadegan, and M. S. Feld, "Coherence properties of red blood cell membrane motions," Phys. Rev. E 76(3), 31902 (2007).

8. Y. Park, M. Diez-Silva, G. Popescu, G. Lykotrafitis, W. Choi, M. S. Feld, and S. Suresh, "Refractive index maps and membrane dynamics of human red blood cells parasitized by plasmodium falciparum," Proc. Natl. Acad. Sci. U.S.A. 105(37), 13730 (2008).

9. D. Szekely, T. Yau, and P. Kuchel, "Human erythrocyte flickering: temperature, ATP concentration, water transport, and cell aging, plus a computer simulation," Eur. Biophys. J. 38(7), 923-939 (2009).

10. B. Rappaz, A. Barbul, A. Hoffmann, D. Boss, R. Korenstein, C. Depeursinge, P. Magistretti, and P. Marquet, "Spatial analysis of erythrocyte membrane fluctuations by digital holographic microscopy," Blood Cells, Mole. Diseases 42(3), 228-232 (2009).

11. M. Costa, I. Ghiran, C. Peng, A. Nicholson-Weller, and A. Goldberger, "Complex dynamics of human red blood cell flickering: alterations with in vivo aging," Phys. Rev. E 78(2), 20901 (2008).

12. G. Popescu, Y. Park, W. Choi, R. Dasari, M. Feld, and K. Badizadegan, "Imaging red blood cell dynamics by quantitative phase microscopy," Blood Cells, Mole. Diseases (2008).

13. T. Browicz, "Further observation of motion phenomena on red blood cells in pathological states," Zbl. med. Wissen 28(1), 625-627 (1890).
14. J. Evans, W. Gratzer, N. Mohandas, K. Parker, and J. Sleep, "Fluctuations of the red blood cell membrane: relation to mechanical properties and lack of ATP dependence," Biophys. J. 94(10), 4134 (2008).

15. S. Tuvia, S. Levin, A. Bitler, and R. Korenstein, "Mechanical fluctuations of the membrane-skeleton are dependent on F-actin ATPase in human erythrocytes," J. Cell Biol. 141(7), 1551-1561 (1998).

16. Y. Park, C. Best, T. Auth, N. Gov, S. Safran, G. Popescu, S. Suresh, and M. Feld, "Metabolic remodeling of the human red blood cell membrane," Proc. Natl. Acad. Sci. U.S.A. 107(4), 1289 (2010).

17. T. Betz, M. Lenz, J. Joanny, and C. Sykes, "ATP-dependent mechanics of red blood cells," Proc. Natl. Acad. Sci. U.S.A. 106(36), 15312-15317 (2009).

18. Y. Park, C. Best, K. Badizadegan, R. Dasari, M. Feld, T. Kuriabova, M. Henle, A. Levine, and G. Popescu, "Measurement of red blood cell mechanics during morphological changes," Proc. Natl. Acad. Sci. U.S.A. 107(15), 6731 (2010).

19. M. Sheetz and S. Singer, "On the mechanism of ATP-induced shape changes in human erythrocyte membranes. I. The role of the spectrin complex," J. Cell Biol. 73(3), 638-646 (1977).

20. D. Tycko, M. Metz, E. Epstein, and A. Grinbaum, "Flow-cytometric light scattering measurement of red blood cell volume and hemoglobin concentration," Appl. Opt. 24(9), 1355-1365 (1985).

21. R. Tishler and F. Carlson, "Quasi-elastic light scattering studies of membrane motion in single red blood cells," Biophys. J. 51(6), 993-997 (1987).

22. M. Amin, Y. Park, N. Lue, R. Dasari, K. Badizadegan, M. Feld, and G. Popescu, "Microrheology of red blood cell membranes using dynamic scattering microscopy," Opt. Express 15(25), 17001-17009 (2007).

23. Y. Park, M. Diez-Silva, D. Fu, G. Popescu, W. Choi, I. Barman, S. Suresh, and M. Feld, "Static and dynamic light scattering of healthy and malaria-parasite invaded red blood cells," J. Biomed. Opt. 15(2), 020506 (2010).

24. N. Boustany, S. Boppart, and V. Backman, "Microscopic imaging and spectroscopy with scattered light," Ann. Rev. Biomed. Eng. 12, 285-314 (2010).

25. H. Ding, E. Berl, Z. Wang, L. Millet, M. Gillette, J. Liu, M. Boppart, and G. Popescu, "Fourier Transform Light Scattering of Biological Structure and Dynamics," Selected Topics in Quantum Electronics, IEEE Journal of 99, 1-10.

26. H. Ding, F. Nguyen, S. A. Boppart, and G. Popescu, "Optical properties of tissues quantified by Fourier transform light scattering" Opt. Lett. 34, 1372 (2009).

27. H. Ding, Z. Wang, F. Nguyen, S. Boppart, and G. Popescu, "Fourier transform light scattering of inhomogeneous and dynamic structures," Phys. Rev. Lett. 101(23), 238102 (2008).

28. H. Ding, L. J. Millet, M. U. Gillette, and G. Popescu, "Actin-driven cell dynamics probed by Fourier transform light scattering," Biomed. Opt. Express 1, 260 (2010).

29. H. Ding, X. Liang, Z. Wang, S. A. Boppart, and G. Popescu, "Tissue scattering parameters from organnell to organ scale," (submitted for publication).

30. G. Popescu, T. Ikeda, R. R. Dasari, and M. S. Feld, "Diffraction phase microscopy for quantifying cell structure and dynamics," Opt. Lett. 31(6), 775-777 (2006).

31. V. Lew, and H. Ferreira, "Calcium transport and the properties of a calcium-activated potassium channel in red cell membranes," in Membrane Properties: Mechanical Aspects, Receptors, Energetics and Calcium-Dependence of Transport, A. K. F. Bronner, Ed., p. 217, Academic Press (1978).

32. Y. K. Park, G. Popescu, K. Badizadegan, R. R. Dasari, and M. S. Feld, "Diffraction phase and fluorescence microscopy," Opt. Express 14(18), 8263-8268 (2006).

33. Y.-K. Park, M. Diez-Silva, G. Popescu, G. Lykotrafitis, W. Choi, M. S. Feld, and S. Suresh, "Refractive index maps and membrane dynamics of human red blood cells parasitized by plasmodium falciparum," Proc. Natl. Acad. Sci. U.S.A. 105(37), 13730 (2008).

34. D. Fu, W. Choi, Y. Sung, S. Oh, Z. Yaqoob, Y. Park, R. Dasari, and M. Feld, "Ultraviolet refractometry using field-based light scattering spectroscopy," Opt. Express 17(21), 18878-18886 (2009). 
35. Y. Park, T. Yamauchi, W. Choi, R. Dasari, and M. Feld, "Spectroscopic phase microscopy for quantifying hemoglobin concentrations in intact red blood cells," Opt. Lett. 34(23), 3668-3670 (2009).

36. W. Choi, C. Yu, C. Fang-Yen, K. Badizadegan, R. Dasari, and M. Feld, "Field-based angle-resolved light-scattering study of single live cells," Opt. Lett. 33(14), 1596-1598 (2008).

37. J. Steinke and A. Shepherd, "Comparison of Mie theory and the light scattering of red blood cells," Appl. Opt. 27(19), 4027-4033 (1988).
38. D. Byrne and J. Earnshaw, "Photon correlation spectroscopy of liquid interfaces. I. Liquid-air interfaces," J. Phys. D: Appl. Phys. 12 (7), 1133-1144 (1979).

39. E. Ainscow, S. Mirshamsi, T. Tang, M. Ashford, and G. Rutter, "Dynamic imaging of free cytosolic ATP concentration during fuel sensing by rat hypothalamic neurones: evidence for ATP-independent control of ATP-sensitive K + channels," J. Physiol. 544(2), 429 (2002).

40. J. D. Wan, W. D. Ristenpart, and H. A. Stone, "Dynamics of shearinduced ATP release from red blood cells," Proc. Nat. Acad. Sci. U.S.A. 105(43), 16432-16437 (2008). 